\title{
Coupling losses in superconducting, torus-shaped wires due to applied magnetic field changes
}

\author{
E.M.J. NIESSEN' ${ }^{\prime}$, L.J.M. VAN DE KLUNDERT ${ }^{\dagger}$, R.M.J. VAN DAMME ${ }^{2}$, \\ F.P.H. VAN BECKUM ${ }^{2}$ and P.J. ZANDBERGEN ${ }^{2}$ \\ Departments of ${ }^{1}$ Applied Physics; ${ }^{2}$ Applied Mathematics; University of Twente, P.O. Box 217 , \\ 7500 AE Enschede, The Netherlands
}

Received 25 March 1991; accepted in revised form 5 November 1991

Key words: superconductors, magnet stability, ac losses

\begin{abstract}
The stationary electric field, current pattern and coupling losses in a multfilamentary, superconducting, twisted, torus-shaped wire are calculated for a torus placed in a homogeneous magnetic field increasing in time at a constant rate and parallel to the torus plane. The radius of the wire is considered to be small compared to the mean radius of the torus. An important parameter for the problem is the ratio between the twist length of the superconducting filaments and the mean radius of the torus. In the configuration considered this parameter is small. The coupling losses are approximately inversely proportional to the square of this ratio. Furthermore, for the wire to have unsaturated parts, the analysis shows that the rate of change of the magnetic field must decrease when this ratio increases.
\end{abstract}

\section{Introduction}

Nowadays, practical use is made of superconductors in large electromagnet systems, e.g., accelerator dipoles in the HERA system at DESY and superconducting coils in tokamak fusion reactors like T-15 in Moscow. The main advantages of using superconducting wires in magnets are a large reduction in power consumption compared to conventional ones as well as the fact that the wires are easier to handle. However, important disadvantages are the much more complicated electrodynamic properties of superconducting wires compared to conventional wires. This is caused by the complicated internal structure of the wire and the non-linear behaviour of some components inside the wire.

A superconducting wire generally consists of many $\left(10^{2}-10^{6}\right)$ filaments of superconducting material embedded in a normal conducting matrix: $\mathrm{Cu}, \mathrm{CuNi}$ or $\mathrm{Al}$. The wire is drawn down to the desired diameter and twisted with a twist length $L_{p}$ in the range 10 to 100 times the wire radius $a$. A cross section of such a wire is given in Fig. 1.

The general problem of calculating the electromagnetic field in a multifilamentary superconducting wire, placed in a time-varying magnetic field, so called a.c. field, has been studied extensively during the last few years $[1,3,4,5]$. As analytical calculations of the field yield only qualitative results, a numerical method was developed $[1,2]$.

Solutions were calculated for simple wire geometries, e.g., straight circular wires in perpendicular [1,3] or parallel [4,5] applied magnetic fields. In important practical applications, however, the wires are used in ring-shaped configurations, e.g., the D-coils in the Next

Deceased. 


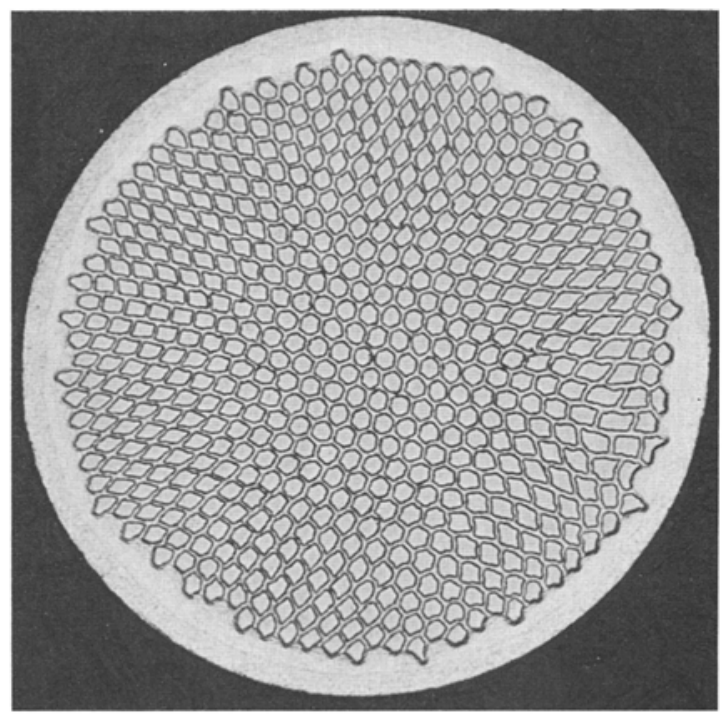

Fig. 1. Cross section of a circular multifilamentary wire.

European Torus fusion reactor [6]. In this article the properties of a D-coil in an a.c. magnetic field are studied, using an ideal torus configuration for the wire.

Here we analytically study the electrodynamic properties of a wire in a ring shape. Of main interest are the dependence on the twist length $L_{p}$, the mean radius of the ring $R_{0}$ and the direction of the applied magnetic field of the generated Ohmic losses (known as coupling losses) in the wire and the current-carrying capacity at low loss levels. The electromagnetic behaviour of the wire can be calculated using Maxwell's equations supplemented with a set of constitutive equations. The constitutive equations give a relation between the electric field and the current density. Furthermore, an appropriate set of initial and boundary conditions must be given. The solution of the problem is not simple, because the constitutive equations are nonlinear (due to the presence of the superconducting filaments) and anisotropic. Both items will be explained in the next section. However, using some simplifications, as we do in this article, we may obtain insight in the behaviour of the wire.

With respect to the ring-shaped wire, we have to consider three stages to be able to describe the complicated spatial geometry. These three stages, describing the multifilamentary twisted torus-shaped wire, are:

1. the description of the superconducting filaments surrounded by normal conducting matrix material;

2. the description of a straight twisted multifilamentary wire;

3. the description of the bent wire in a torus shape.

In the next section we will give a detailed description of every stage considered.

We now focus on the magnetic field aspects. The applied time-dependent magnetic field is considered to be uniform in space and parallel to the plane of the torus with constant time derivative $\dot{B}^{A}$. This field, chosen in the $z$ direction (see Fig. 2) $\left(\dot{B}_{z}^{A}\right)$ will be perpendicular to the wire for coordinates $(x, z)=\left(0, \pm R_{0}\right)$ and parallel for $(x, z)=\left( \pm R_{0}, 0\right)$. For other coordinates $(x, z)$ on the wire the field is partly perpendicular and partly parallel.

We consider the centre of the wire to be unsaturated, which restricts the value of $\left|\dot{B}_{z}^{A}\right|$ as 


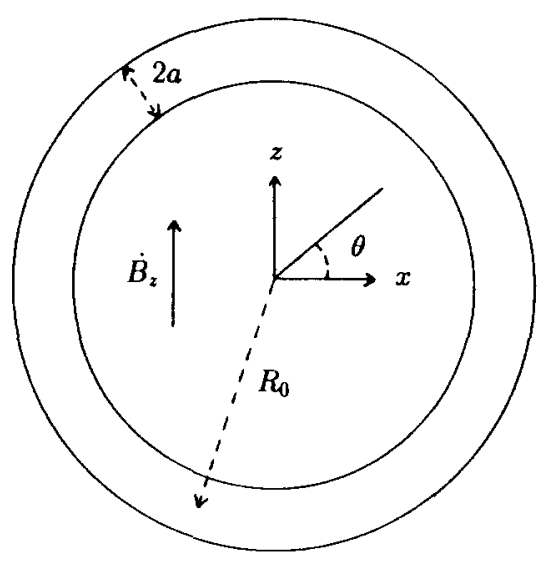

Fig. 2. Circular configuration for the wire with radius $a$ in a torus shape with mean radius $R_{0}$. Here $a \ll R_{0}$.

we will show. For $\dot{B}_{z}^{A}$ is constant, we calculate the stationary solution for the electric field and the currents. Furthermore, we calculate the coupling losses due to the Ohmic dissipation of the matrix material. The currents in the unsaturated superconducting filaments are non-dissipative.

The electric field in a $\mathrm{D}$-coil for a realistic field configuration can be calculated for the unsaturated case using the superposition principle. As long as the wire is unsaturated, the problem of calculating the electric field and currents is linear. This means that we write the applied magnetic field as a Fourier series and calculate the corresponding electric field terms. Addition of all the electric field terms gives the resulting electric field, which can be used for calculating the coupling losses. Note that this calculation method can only be used as long as the problem is linear, i.e. the partly saturated solution, where the nonlinear behaviour of the superconducting filaments must be taken into account, cannot be analyzed this way and therefore we will not consider this case.

Calculations with respect to this configuration can also be performed approximating the problem by considering a straight wire and applying a spatially periodic time-dependent magnetic field. We will compare these results with our exact solution.

\section{The spatial geometry}

In this section we give a detailed description of the geometry of all three stages introduced in the previous section.

In stage 1 we investigate the interior of the wire on the smallest scale: we consider superconducting filaments surrounded by normal conducting matrix material. The superconducting behaviour of the filaments is described by the critical state model $[7,8]$. According to this model the relation between the local superconducting current density in the filament $j_{\|}^{s}$ and the electric field parallel to the filament $E_{\|}$can be written for zero filament radius as

$$
j_{\|}^{s} \begin{cases}\in\left(-j_{c}, j_{c}\right) & \text { if } E_{\|}=0 \\ =j_{c} \operatorname{sign}\left(E_{\|}\right) & \text {if } E_{\|} \neq 0,\end{cases}
$$

where $j_{c}$ is the critical current density. $E_{\|}=0$ means that the filament is unsaturated, while 
$E_{\|} \neq 0$ holds for a saturated filament. The conductivity of the normal (Ohmic) conducting material is $\sigma$.

In stage 2 we regard a scale considerably larger than stage 1, which means that we get a more macroscopic view. Here we consider a straight multifilamentary wire with circular cross section. The radius of the wire is $a$. We consider the central axis of the wire parallel to the $z$ direction of the cylindrical coordinate system. The wire is twisted with twistlength $L_{p}$. Part of the path of two twisted filaments in a straight wire is shown in Fig. 3. We choose a coordinate system with the direction parallel to the filaments, the radial direction and the direction defined by the outer product of the two aforementioned directions, denoted by the perpendicular symbol: $\perp$. Recognize that both the $r$ direction and the $\perp$ direction are perpendicular to the filaments. The constitutive equations for an unsaturated wire are given in this coordinate system by

$$
\left(j_{r}, j_{\perp}, j_{\|}\right)=\left(\sigma_{\perp} E_{r}, \sigma_{\perp} E_{\perp}, \lambda j_{\|}^{s}+\sigma_{\|} E_{\|}\right)
$$

with

$$
\begin{gathered}
\sigma_{\perp}=\frac{1-\lambda}{1+\lambda} \sigma, \\
\sigma_{\|}=(1-\lambda) \sigma,
\end{gathered}
$$

with $\lambda$ the fraction of superconducting material. Notice that in the normal conducting conductivity matrix in this coordinate system only the principal diagonal elements can be non-zero.

We can calculate the constitutive equations in the cylindrical $r, \phi, z$ coordinate system using the following transformations:

$$
\begin{aligned}
\mathbf{e}_{\perp} & =\cos \psi \mathbf{e}_{\phi}-\sin \psi \mathbf{e}_{z}, \\
\mathbf{e}_{\|} & =\sin \psi \mathbf{e}_{\phi}+\cos \psi \mathbf{e}_{z},
\end{aligned}
$$

with

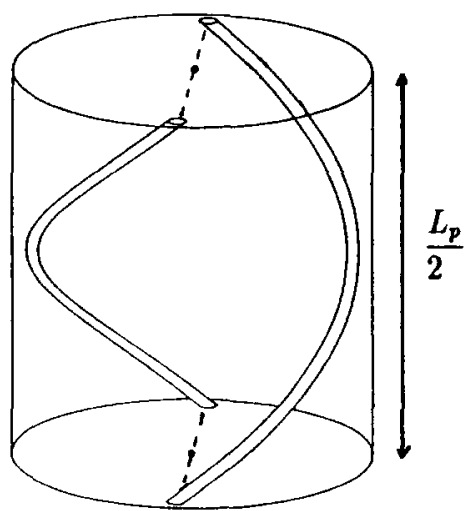

Fig. 3. Schematic view of the path of two twisted filaments in a straight wire. 


$$
\tan \psi=\frac{2 \pi r}{L_{p}}
$$

This gives for the constitutive equations for an unsaturated wire

$$
\left(j_{r}, j_{\phi}, j_{z}\right)=\left(\sigma_{\perp} E_{r}, \lambda j_{\|}^{s} \sin \psi+\sigma_{\perp} E_{\phi}, \lambda j_{\|}^{s} \cos \psi+\sigma_{\perp} E_{z}\right) .
$$

The first terms of $j_{\phi}$ and $j_{z}$ result from the superconducting current density of the filaments while the second term is just an Ohmic term resulting from the matrix material. In the normal conducting conductivity matrix we now also have off diagonal elements that can be non-zero. Notice that the current density in the $r$ direction has no superconducting term because the parallel direction is perpendicular to the $r$ direction. In case the wire is saturated, $j_{\phi}$ and $j_{z}$ contain an extra term due to the fact that $E_{\|} \neq 0$. This extra term is not given in Eq. (8).

Recognize that $\psi$ is a function of $r$ only and that the trajectory of a filament is given by

$$
z=\frac{L_{p}}{2 \pi} \phi+z_{0}
$$

with $z_{0}$ a constant.

In Carr's approach [9] the electromagnetic field and current density are averaged over an area which is large compared to the microstructure and small compared to the macroscopic view. Carr showed that the Maxwell equations hold for a multifilamentary superconductor when the electromagnetic quantities are averaged over a volume with dimensions of the order of a filament cross section.

In the last stage, we investigate the largest scale and consider the bent wire in a torus configuration with radius of the central axis $R_{0}$. Note that this bending affects the path of every filament in the wire, except the one along the central axis. The path of one filament in this torus shaped, twisted wire is shown in Fig. 4.

We want to perform the calculations in the natural $r, \phi, \theta$ system where $\theta$ represents the angle on the torus with the positive $x$-axis and $r$ and $\phi$ are the cylindrical coordinates perpendicular to $\theta$. The configuration and definitions are outlined in Fig. 2. $x, y, z$ expressed in $r, \phi, \theta$ is given in Eq. (20) of the next section.

Before we can give the constitutive equations for this bent wire, we need to know the trajectory of a twisted filament in the bent wire. The constitutive equations for a straight wire are previously described (stage 2). When we start with this configuration we see that due to the bending of the wire the parallel direction is changed and becomes a function of $r$, $\phi$ and $\theta$. The stress parameters in the wire will influence the resulting parallel direction after

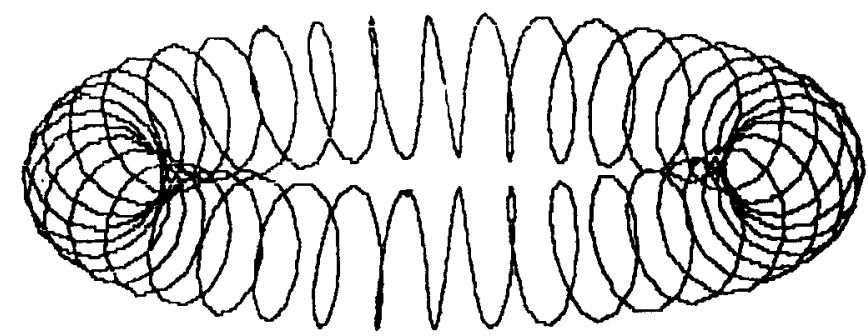

Fig. 4. Schematic view of the path of a twisted filament in the torus shaped wire. 
bending. We do not include these phenomena in the analysis and assume purely geometrical deformation resulting in

$$
\mathbf{e}_{\|}=\sin \psi \mathbf{e}_{\phi}+\cos \psi \mathbf{e}_{\theta},
$$

with $\tan \psi$ given by Eq. (7), to calculate the trajectory of a filament fulfilling this condition. Now we calculate the $\phi(\theta)$ dependence in case of this assumption when $r$ is not a function of $\theta$. This means that we fictitiously adapt the twisting of the filaments in such a way that the angle $\psi$ is not a function of $\phi$ and $\theta$. A (not normalized) vector in the $\|$ direction (tangent to the filament) $\mathbf{p}_{\|}$is found as the derivative of the position vector: $x \mathbf{e}_{x}+y \mathbf{e}_{y}+z \mathbf{e}_{z}$ along the filament, i.e. $\phi=\phi(\theta)$ and $r$ is constant:

$$
\begin{aligned}
\mathbf{p}_{\|} & =\left[\partial_{\theta} x+\partial_{\phi} x \mathrm{~d}_{\theta} \phi\right] \mathbf{e}_{x}+\left[\partial_{\theta} y+\partial_{\phi} y \mathrm{~d}_{\theta} \phi\right] \mathbf{e}_{y}+\left[\partial_{\theta} z+\partial_{\phi} z \mathrm{~d}_{\theta} \phi\right] \mathbf{e}_{z} \\
& =c_{1}\left(r, \theta, \phi(\theta), \mathrm{d}_{\theta} \phi\right) \mathbf{e}_{r}+c_{2}\left(r, \theta, \phi(\theta), \mathrm{d}_{\theta} \phi\right) \mathbf{e}_{\phi}+c_{3}\left(r, \theta, \phi(\theta), \mathrm{d}_{\theta} \phi\right) \mathbf{e}_{\theta} .
\end{aligned}
$$

In order to satisfy Eq. (10) we obtain the requirements:

$$
c_{1} \equiv 0 \quad \text { and } \quad \frac{c_{2}}{c_{3}} \equiv \tan \psi=\frac{2 \pi r}{L_{p}},
$$

which gives:

$$
c_{1} \equiv 0 \quad \text { and } \quad \mathrm{d}_{\theta} \phi=\frac{2 \pi}{L_{p}}\left(R_{0}+r \cos \phi\right)
$$

with solution:

$$
\phi=2 \arctan \left[\frac{\sqrt{R_{0}^{2}-r^{2}}}{R_{0}-r} \tan \left(\frac{\theta \pi \sqrt{R_{0}^{2}-r^{2}}}{L_{p}}\right)\right]+\phi_{0}
$$

and inverse:

$$
\theta=\frac{L_{p}}{\pi \sqrt{R_{0}^{2}-r^{2}}} \arctan \left[\frac{\left(R_{0}-r\right) \tan \phi / 2}{\sqrt{R_{0}^{2}-r^{2}}}\right]+\theta_{0} .
$$

Notice that the important periodicity parameter is $L_{p} /\left(2 \pi \sqrt{R_{0}^{2}-r^{2}}\right)$ and for $R_{0} \gg r$ we see that the trajectory of a filament is given by

$$
\theta=\frac{L_{p}}{2 \pi R_{0}} \phi+\theta_{0} \equiv k \phi+\theta_{0}
$$

with $\theta_{0}$ a constant. When we compare this result with eq. (9) we see that for $R_{0} \gg r$ the twist is not influenced by the bending of the wire.

Now the constitutive equations read:

$$
\left(j_{r}, j_{\phi}, j_{\theta}\right)=\left(\sigma_{\perp} E_{r}, \tan \psi j_{0}+\sigma_{\perp} E_{\phi}, j_{0}+\sigma_{\perp} E_{\theta}\right)
$$

with

$$
j_{0}=\lambda j_{\|}^{s} \cos \psi
$$


Summarizing we can say that the geometry is fully determined by three parameters: $a, L_{p}$, and $R_{0}$. We calculate the electric field and the currents in case $a$ is very small compared to $R_{0}$ and $L_{p}$ is smaller than $2 \pi R_{0}$. We consider $k \equiv L_{p} /\left(2 \pi R_{0}\right)$ an irrational number. This means that the path of a filament is never closed so there exists no superconducting closed path. A consequence of this assumption is that no resonance appears in the system. This noresonance condition will be explained in the next sections. Note that the shown closed path of a filament in Fig. 4 does not satisfy this condition.

\section{Vector analysis in the toroidal coordinate system}

In this section we describe the important calculus in the toroidal coordinate system [10]. The $\left(u_{1}, u_{2}, u_{3}\right) \equiv(r, \phi, \theta)$ system is a positively oriented orthogonal coordinate system. The coordinate transformation for the vector $\mathbf{x}$ with $x, y$ and $z$ coordinates to the new coordinate system reads

$$
(x, y, z)=\left(\left(R_{0}+r \cos \phi\right) \cos \theta, r \sin \phi,\left(R_{0}+r \cos \phi\right) \sin \theta\right) .
$$

The infinitesimal arc length and volume are given by:

$$
\begin{aligned}
& \mathrm{d} l^{2}=h_{1}^{2} \cdot \mathrm{d} u_{1}^{2}+h_{2}^{2} \mathrm{~d} u_{2}^{2}+h_{3}^{2} \mathrm{~d} u_{3}^{2}, \\
& \mathrm{~d} V=h_{1} h_{2} h_{3} \mathrm{~d} u_{1} \mathrm{~d} u_{2} \mathrm{~d} u_{3},
\end{aligned}
$$

respectively. We calculate $h_{i}$ using:

$$
h_{i}^{2}=\left(\frac{\partial x}{\partial u_{i}}\right)^{2}+\left(\frac{\partial y}{\partial u_{i}}\right)^{2}+\left(\frac{\partial z}{\partial u_{i}}\right)^{2}
$$

which gives for the elementary lengths:

$$
\begin{aligned}
& h_{1}=h_{r}=1, \\
& h_{2}=h_{\phi}=r, \\
& h_{3}=h_{\theta}=R_{0}+r \cos \phi .
\end{aligned}
$$

The unit vectors in this coordinate system are given by:

$$
\mathbf{e}_{i}=\frac{1}{h_{i}} \frac{\partial \mathbf{x}}{\partial u_{i}}
$$

and in explicit notation:

$$
\begin{aligned}
& \mathbf{e}_{r}=\cos \phi \cos \theta \mathbf{e}_{x}+\sin \phi \mathbf{e}_{y}+\cos \phi \sin \theta \mathbf{e}_{z}, \\
& \mathbf{e}_{\phi}=-\sin \phi \cos \theta \mathbf{e}_{x}+\cos \phi \mathbf{e}_{y}-\sin \phi \sin \theta \mathbf{e}_{z}, \\
& \mathbf{e}_{\theta}=-\sin \theta \mathbf{e}_{x}+\cos \theta \mathbf{e}_{z} .
\end{aligned}
$$


The inverse transformation can easily be derived. Some important vector operations on a vector read:

$$
\begin{aligned}
& (\boldsymbol{\nabla} \times \mathbf{A})_{1}=\frac{1}{h_{2} h_{3}}\left[\frac{\partial}{\partial u_{2}}\left(h_{3} A_{3}\right)-\frac{\partial}{\partial u_{3}}\left(h_{2} A_{2}\right)\right], \\
& \boldsymbol{\nabla} \cdot \mathbf{A}=\frac{1}{h_{1} h_{2} h_{3}} \sum_{i} \frac{\partial}{\partial u_{i}}\left(h_{1} h_{2} h_{3} A_{i} / h_{i}\right) .
\end{aligned}
$$

The remaining components of $(\boldsymbol{\nabla} \times \mathbf{A})$ are obtained by cyclic interchanges of the suffixes.

\section{Definition of the problem}

Using the results of the previous section we can derive the set of equations describing the problem in the $r, \phi, \theta$ coordinate system.

The Maxwell equations in case of a stationary problem read

$$
\begin{aligned}
& (\boldsymbol{\nabla} \times \mathbf{E})_{r}=\frac{1}{r} \partial_{\phi} E_{\theta}-\frac{1}{R_{0}+r \cos \phi}\left[\partial_{\theta} E_{\phi}+\sin \phi E_{\theta}\right]=-\dot{B}_{r}^{A}, \\
& (\boldsymbol{\nabla} \times \mathbf{E})_{\phi}=-\partial_{r} E_{\theta}-\frac{1}{R_{0}+r \cos \phi}\left[-\partial_{\theta} E_{r}+\cos \phi E_{\theta}\right]=-\dot{B}_{\phi}^{A}, \\
& (\boldsymbol{\nabla} \times \mathbf{E})_{\theta}=\frac{1}{r}\left[\partial_{r}\left(r E_{\phi}\right)-\partial_{\phi} E_{r}\right]=-\dot{B}_{\theta}^{A} .
\end{aligned}
$$

The applied magnetic field $\dot{B}_{z}^{A}$ in the $z$ direction can be written in its $r, \phi, \theta$ components:

$$
\dot{\mathbf{B}}^{A}=\dot{B}_{z}^{A}(\cos \phi \sin \theta,-\sin \phi \sin \theta, \cos \theta) \text {. }
$$

Conservation of the bulk current density $\mathbf{j}$ in the interior of the wire can be written as

$$
\boldsymbol{\nabla} \cdot \mathbf{j}=\frac{1}{r}\left[\partial_{r}\left(r j_{r}\right)+\partial_{\phi} j_{\phi}\right]+\frac{1}{R_{0}+r \cos \phi}\left[\cos \phi j_{r}-\sin \phi j_{\phi}+\partial_{\theta} j_{\theta}\right]=0 \text {. }
$$

At the surface of the wire $r=a$ we consider a surface current with components $J_{\phi}$ and $J_{\theta}$ in the $\phi$ and $\theta$ direction respectively. From the fact that no current flows out of the wire at the boundary $r=a$, conservation of current can be written as

$$
-\left(R_{0}+a \cos \phi\right) a j_{r}(r=a)+\left(R_{0}+a \cos \phi\right) \partial_{\phi} J_{\phi}-J_{\phi} a \sin \phi+a \partial_{\theta} J_{\theta}=0 .
$$

We consider the surface current at $r=a$ to flow in the direction of the filaments, i.e.

$$
\left(J_{\phi}, J_{\theta}\right)=\left(\sin \psi_{a} J_{\|}, \cos \psi_{a} J_{\|}\right)
$$

with

$$
\tan \psi_{a}=\frac{2 \pi a}{L_{p}}
$$

In case the interior of the wire is unsaturated, i.e. $E_{\|}=0$, we obtain 


$$
E_{\theta}=-\tan \psi E_{\phi}
$$

The boundary conditions can easily be formulated: the electric field and currents are periodical functions of $\phi$ and $\theta$ with period length $2 \pi n$ with $n=1,2,3, \ldots$; the electric field and currents are bounded for $r=0$ and no current flows out of the wire at $r=a$.

Using the constitutive equations we see that we have to solve 4 unknowns: $E_{\phi}, E_{r}, j_{0}$ and $J_{\|}$for which we use the Eqs $(33),(34+35),(37)$ and $(38)$. The calculations are outlined in the next section.

\section{Calculation of the electric field and currents}

In this section we calculate the electric field components and the currents using the set of equations described in the previous section. The differential equations cannot be solved directly, so we use a perturbation series in $r$. This is legitimate as $a \ll R_{0}$. We calculate a first order accurate solution for the case that no resonances appear in the system, i.e. $k$ is an irrational number. The calculations of all 4 unknowns $E_{\phi}, E_{r}, j_{0}$ and $J_{\|}$are identical except for the implementation of the boundary conditions. We show the calculation of $E_{\phi}$ in detail and summarize the calculations of the other 3 unknowns.

Substituting Eq. (41) and (7) in (33) gives:

$$
2 \pi \partial_{\phi} E_{\phi}+\frac{L_{p}}{R_{0}} \partial_{\theta} E_{\phi}=L_{p} \dot{B}_{r}^{A}+\frac{r}{R_{0}}\left[L_{p} \cos \phi \dot{B}_{r}^{A}+2 \pi \sin \phi E_{\phi}-2 \pi \cos \phi \partial_{\phi} E_{\phi}\right]
$$

Observe that $E_{\phi}$ is the only unknown in this equation. The last term in this equation is small compared to the first one for $a \ll R_{0}$. The one but last term is considered to be small. This assumption must be checked later. Then the homogeneous equation

$$
2 \pi \partial_{\phi} E_{\phi}+\frac{L_{p}}{R_{0}} \partial_{\theta} E_{\phi}=0
$$

has the solution

$$
E_{\phi}^{\mathrm{Hom}}=A(r) F(\theta / k-\phi)
$$

where $F$ is any (smooth enough) function with argument $(\theta / k-\phi)$. The solution has to be a periodic function of $\phi$ and $\theta$ with period length $2 \pi n$. As $k$ is irrational, the periodicity in $\phi$ and $\theta$ imposes $F$ to be a constant. Furthermore, using the boundedness of $E_{r}$, one can easily show with eq. (35) that $A(r)$ must be zero. Together this results in: $E_{\phi}^{\mathrm{Hom}} \equiv 0$. This explains what we mean by 'no resonances appear in the system': now there has to be an applied field to obtain a non-zero electric field and non-zero currents.

We now calculate the particular solution of Eq. (42). For this purpose we write $E_{\phi}$ as a power series in $r$ :

$$
E_{\phi}=E_{\phi}^{(0)}+r E_{\phi}^{(1)}+r^{2} E_{\phi}^{(2)}+\cdots
$$

This gives 


$$
\begin{aligned}
& 2 \pi \partial_{\phi} E_{\phi}^{(0)}+\frac{L_{p}}{R_{0}} \partial_{\theta} E_{\phi}^{(0)}=L_{p} \dot{B}_{r}^{A}, \\
& 2 \pi \partial_{\phi} E_{\phi}^{(1)}+\frac{L_{p}}{R_{0}} \partial_{\theta} E_{\phi}^{(1)}=\frac{L_{p}}{R_{0}} \cos \phi \dot{B}_{r}^{A}+\frac{2 \pi}{R_{0}} \sin \phi E_{\phi}^{(0)}-\frac{2 \pi}{R_{0}} \cos \phi \partial_{\phi} E_{\phi}^{(0)}
\end{aligned}
$$

for $E_{\phi}^{(0)}$ and $E_{\phi}^{(1)}$ respectively. The solution is

$$
\begin{aligned}
& E_{\phi}^{(0)}=\frac{L_{p} \dot{B}_{z}^{A}}{2 \pi\left(1-k^{2}\right)}[\sin \phi \sin \theta+k \cos \phi \cos \theta], \\
& E_{\phi}^{(1)}=-\frac{\dot{B}_{z}^{A}}{2} \cos \theta-\frac{\dot{B}_{z}^{A} k\left(1+2 k^{2}\right)}{\left(1-k^{2}\right)\left(4-k^{2}\right)} \sin 2 \phi \sin \theta-\frac{\dot{B}_{z}^{A} k^{2}\left(5+k^{2}\right)}{2\left(1-k^{2}\right)\left(4-k^{2}\right)} \cos 2 \phi \cos \theta .
\end{aligned}
$$

Recognize that the one but last term of eq. (42) indeed is small compared to the left hand side for $a \ll R_{0}$, which was assumed. The higher order Fourier terms in $\phi$ appear because $h_{\theta}$ contains the term $r \cos \phi$, see Eq. (26).

We obtain an equation for $E_{r}$ similar to the equation for $E_{\phi}$ by adding Eq. (34) and (35). It contains terms $E_{\phi}$, which is known. The homogeneous solution is zero, for reasons similarly applying to $E_{\phi}$, except for a possible term which is only a function of $r$. This term being non-zero would give rise to an effective current into or out of a torus shell, which is forbidden.

The expansion of $E_{r}$ in powers of $r$ results in

$$
\begin{aligned}
E_{r}^{(0)}= & \frac{L_{p} \dot{B}_{z}^{A}}{2 \pi\left(1-k^{2}\right)}[-\cos \phi \sin \theta+k \sin \phi \cos \theta], \\
E_{r}^{(1)}= & \frac{\dot{B}_{z}^{A}\left(4-9 k^{2}+2 k^{4}\right)}{k\left(1-k^{2}\right)\left(4-k^{2}\right)} \sin \theta+\frac{\dot{B}_{z}^{A} k\left(1+2 k^{2}\right)}{\left(1-k^{2}\right)\left(4-k^{2}\right)} \cos 2 \phi \sin \theta \\
& -\frac{\dot{B}_{z}^{A} k^{2}\left(5+k^{2}\right)}{2\left(1-k^{2}\right)\left(4-k^{2}\right)} \sin 2 \phi \cos \theta .
\end{aligned}
$$

$j_{0}$ is calculated using Eq. (37):

$$
\begin{aligned}
2 \pi \partial_{\phi} j_{0}+\frac{L_{p}}{R_{0}} \partial_{\theta} j_{0}= & -\frac{L_{p}}{R_{0}}\left(\cos \phi+\frac{R_{0}}{r}\right)\left(\partial_{r} r j_{r}+\partial_{\phi} \sigma_{\perp} E_{\phi}\right)-\frac{L_{p}}{R_{0}} \cos \phi j_{r}+\frac{L_{p}}{R_{0}} \sin \phi \sigma_{\perp} E_{\phi} \\
& +\frac{2 \pi r}{R_{0}}\left[\partial_{\theta} \sigma_{\perp} E_{\phi}+\sin \phi j_{0}-\cos \phi \partial_{\phi} j_{0}\right] .
\end{aligned}
$$

The homogeneous solution is zero by repeating the same argument, for $E_{\phi}$, except for the term which is only a function of $r$. This term is excluded however, because a periodic field results in periodic currents. The expansion of $j_{0}$ in powers of $r$ results in

$$
j_{0}^{(0)}=\frac{\sigma_{\perp} \dot{B}_{z}^{A} L_{p}}{2 \pi} \frac{\left(2-5 k^{2}\right)}{k^{2}\left(1-k^{2}\right)} \cos \theta
$$

$j_{0}^{(1)}$ is not calculated because we need $E_{\phi}^{(2)}$ and $E_{r}^{(2)}$ which are not calculated. For the calculation of the surface current we use Eq. (38): 
$-\sigma_{\perp}\left(R_{0}+a \cos \phi\right) a E_{r}(r=a)+\left(R_{0}+a \cos \phi\right) \sin \psi_{a} \partial_{\phi} J_{\|}$

$-a \sin \phi \sin \psi_{a} J_{\|}+a \cos \psi_{a} \partial_{\theta} J_{\theta}=0$.

Using the consistent approximations

$$
\begin{aligned}
& \cos \psi_{a}=1-\frac{1}{2}\left(\frac{2 \pi a}{L_{p}}\right)^{2}+O\left(\left(\frac{a}{L_{p}}\right)^{4}\right), \\
& \sin \psi_{a}=\frac{2 \pi a}{L_{p}}+O\left(\left(\frac{a}{L_{p}}\right)^{3}\right),
\end{aligned}
$$

we obtain

$$
\begin{aligned}
2 \pi \partial_{\phi} J_{\|}+\frac{L_{p}}{R_{0}} \partial_{\theta} J_{\|}= & L_{p} \sigma_{\perp} E_{r}(r=a) \\
& +\frac{a}{R_{0}}\left[L_{p} \cos \phi \sigma_{\perp} E_{r}(r=a)+2 \pi \sin \phi J_{\|}-2 \pi \cos \phi \partial_{\phi} J_{\|}\right] \\
& +\frac{L_{p}}{2 R_{0}}\left(\frac{2 \pi a}{L_{p}}\right)^{2} \partial_{\theta} J_{\|}+O\left(\left(\frac{a}{L_{p}}\right)^{s}\right)
\end{aligned}
$$

Again the homogeneous solution vanishes and the expansion of $J_{\|}$in powers of $r$ results in

$$
\begin{aligned}
J_{\|}^{(0)}= & -\sigma_{\perp} \dot{B}_{z}^{A}\left(\frac{L_{p}}{2 \pi}\right)^{2}\left[\frac{1+k^{2}}{(k+1)^{2}(k-1)^{2}} \sin \phi \sin \theta+\frac{2 k}{(k+1)^{2}(k-1)^{2}} \cos \phi \cos \theta\right] \\
J_{\|}^{(1)}= & \frac{\sigma_{\perp} \dot{B}_{z}^{A} L_{p}}{2 \pi}\left[\frac{2-5 k^{2}}{2 k^{2}(k+1)(k-1)} \cos \theta+\frac{k\left(12+43 k^{2}-17 k^{4}-2 k^{6}\right)}{2(k+2)^{2}(k+1)^{2}(k-1)^{2}(k-2)^{2}} \sin 2 \phi \sin \theta\right] \\
& +\frac{\sigma_{\perp} \dot{B}_{z}^{A} L_{p}}{2 \pi} \frac{k^{2}\left(40+7 k^{2}-11 k^{4}\right)}{2(k+2)^{2}(k+1)^{2}(k-1)^{2}(k-2)^{2}} \cos 2 \phi \cos \theta
\end{aligned}
$$

We can check the expressions for the bulk and surface current by calculating the total current through a cross section: $\theta$ is constant. $j_{\theta}$ and $J_{\theta}$ must, in any order of $a$, fulfil the equation

$$
\int_{-\pi}^{\pi} J_{\theta} a \mathrm{~d} \phi+\int_{-\pi}^{\pi} \int_{0}^{a} j_{\theta} r \mathrm{~d} \phi \mathrm{d} r=0
$$

The first and second order expressions in $a$ are

$$
\begin{aligned}
& \int_{-\pi}^{\pi} J_{\|}^{(0)} \cos \psi_{a} a \mathrm{~d} \phi=0 \\
& \int_{-\pi}^{\pi} J_{\theta}^{(1)} a^{2} \mathrm{~d} \phi+\int_{-\pi}^{\pi} \int_{0}^{a} j_{\theta}^{(0)} r \mathrm{~d} \phi \mathrm{d} r=\int_{-\pi}^{\pi} J_{\|}^{(1)} \cos \psi_{a} a^{2} \mathrm{~d} \phi+\int_{-\pi}^{\pi} \int_{0}^{a} j_{\theta}^{(0)} r \mathrm{~d} \phi \mathrm{d} r \\
& =\int_{-\pi}^{\pi} J_{\|}^{(1)} a^{2} \mathrm{~d} \phi+\int_{-\pi}^{\pi} \int_{0}^{a} j_{0}^{(0)} r \mathrm{~d} \phi \mathrm{d} r+O\left(a^{3}\right) \\
& =0+O\left(a^{3}\right) \text {, }
\end{aligned}
$$


which means that up to the order of calculation of the expressions, conservation of current is fulfilled. The surface current through the stated plane is, second order accurate in $a$

$$
\int_{-\pi}^{\pi} J_{\|}^{(1)} a^{2} \mathrm{~d} \phi=\sigma_{\perp} \dot{B}_{z}^{A} L_{p} a^{2} \frac{\left(2-5 k^{2}\right)}{2 k^{2}\left(k^{2}-1\right)} \cos \theta .
$$

\section{Interpretation}

In this section we will discuss the consequences of the solution derived in the previous section. We calculate an upper bound on $\left|\dot{B}_{z}^{A}\right|$ for the centre of the wire to be unsaturated. Furthermore we calculate the coupling loss power density for the unsaturated wire and we give an interpretation of the expressions in case $k$ is small. We will also discuss the assumption of a stationary solution.

The maximum value of $\left|\dot{B}_{z}^{A}\right|$ for which the centre of the wire is unsaturated can be calculated using

$$
\left|j_{\|}^{s}\right|_{r=0}=\left|\frac{j_{0}}{\lambda \cos \psi}\right|_{r=0}<j_{c} .
$$

For small values of $k$ this gives:

$$
\left|\dot{B}_{z}^{A}\right|<\frac{\lambda j_{c} L_{p}}{4 \pi R_{0}^{2} \sigma_{\perp}} .
$$

Notice that $\left|\dot{B}_{z}^{A}\right|$ must be very small for great values of $R_{0}$ for the wire to be unsaturated. Due to the shielding currents the maximal transport current at low losses $\left(I_{\max }\right)$ is for small values of $k$ approximated by

$$
I_{\max }=\pi a^{2}\left[\lambda j_{c}-2 \sigma_{\perp}\left|\dot{B}_{z}^{A}\right| \frac{L_{p}}{2 \pi k^{2}}\right] .
$$

The loss power density in the unsaturated case reads:

$$
\begin{aligned}
P / \mathrm{Vol}= & \frac{\sigma_{\perp}}{\mathrm{Vol}} \int_{-\pi}^{\pi} \int_{-\pi}^{\pi} \int_{0}^{a}\left(E_{\perp}^{2}+E_{r}^{2}\right)\left(R_{0}+r \cos \phi\right) r \mathrm{~d} \theta \mathrm{d} \phi \mathrm{d} r \\
= & \frac{32-168 k^{2}+232 k^{4}-79 k^{6}+16 k^{8}+3 k^{10}}{8 k^{2}\left(1-k^{2}\right)^{2}\left(4-k^{2}\right)^{2}} a^{2}\left(\dot{B}_{z}^{A}\right)^{2} \sigma_{\perp} \\
& +\frac{64+32 k^{2}-28 k^{4}+4 k^{6}}{8\left(1-k^{2}\right)^{2}\left(4-k^{2}\right)^{2}} \frac{L_{p}^{2}}{4 \pi^{2}}\left(\dot{B}_{z}^{A}\right)^{2} \sigma_{\perp} \quad\left[\mathrm{W} / \mathrm{m}^{3}\right],
\end{aligned}
$$

with

$$
\begin{aligned}
& \mathrm{Vol}=2 \pi R_{0} a^{2} \\
& E_{\perp}=E_{\phi}^{(0)}+r E_{\phi}^{(1)} \text { and } E_{r}=E_{r}^{(0)}+r E_{r}^{(1)} .
\end{aligned}
$$


The first and second term of Eq. (68) are due to the parallel and perpendicular component of the applied field $\dot{B}_{z}^{A}$, respectively.

The expressions for $k$ small (here $L_{p}$ is constant and $R_{0}$ is large) for the electric field components, the surface current, the bulk current density and the coupling loss power densitv are

$$
\begin{aligned}
& E_{r}=-\frac{\dot{B}_{z}^{A} L_{p}}{2 \pi} \cos \phi \sin \theta+\frac{\dot{B}_{z}^{A} r}{k} \sin \theta+O\left(r^{2}\right) \\
& E_{\phi}=\frac{\dot{B}_{z}^{A} L_{p}}{2 \pi} \sin \phi \sin \theta-\frac{\dot{B}_{z}^{A} r}{2} \cos \theta+O\left(r^{2}\right) \\
& E_{\theta}=-\frac{2 \pi r}{L_{p}} E_{\phi} \\
& J_{\|}=-\sigma_{\perp} \dot{B}_{z}^{A}\left(\frac{L_{p}}{2 \pi}\right)^{2} \sin \phi \sin \theta-\sigma_{\perp} \frac{\dot{B}_{z}^{A} L_{p}}{2 \pi} \frac{a}{k^{2}} \cos \theta+O\left(a^{2}\right), \\
& j_{0}=\sigma_{\perp} \frac{\dot{B}_{z}^{A} L_{p}}{\pi} \frac{1}{k^{2}} \cos \theta+O(r), \\
& P / \mathrm{Vol}=\frac{\sigma_{\perp}\left(\dot{B}_{z}^{A}\right)^{2}}{2}\left(\frac{L_{p}}{2 \pi}\right)^{2}+\frac{\sigma_{\perp}\left(\dot{B}_{z}^{A}\right)^{2}}{4} \frac{a^{2}}{k^{2}}+O\left(a^{3}\right) .
\end{aligned}
$$

These relations are depicted in Fig. 5 for $\phi=\pi / 4$ and parameter setting: $a=0.015 \mathrm{~m}$, $L_{p}=0.43 \mathrm{~m}$ and $R_{0}=7 \mathrm{~m}$. Every field component is scaled on its own maximal value. $E_{r}$ and $J_{\|}$consist mainly of the parallel field term (the second term in Eqs (71) and (74)). In $E_{\phi}$ and $E_{\theta}$ mainly the perpendicular term is present (the first term in Eq. (72)). The parallel field term has a small influence because $E_{\phi}(\theta=0)$ is non zero. In Fig. $6 E_{\phi}$ scaled on the maximum value is given as function of $\theta$ for several values of $\phi$. The parameter setting is not changed. As can be seen, $E_{\phi}$ changes sign in every cross section for this parameter setting.

Recognize that only $E_{\phi}$ is bounded for $k \rightarrow 0$. The singularity of $E_{r}$ and the currents can be understood as follows: a $\dot{B}^{A}$ component parallel to the axis of a twisted wire causes a bulk current to flow in the direction of the magnetic field and a surface current in the opposite

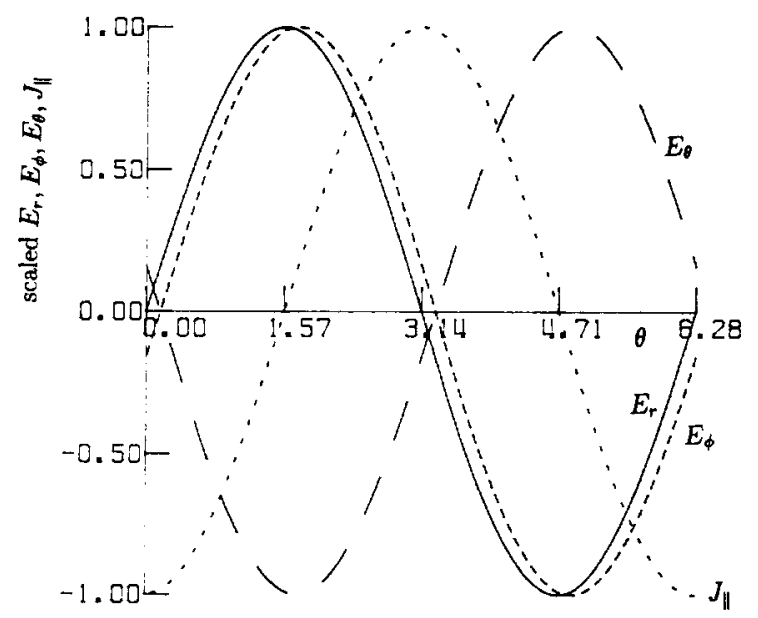

Fig. 5. Scaled value of $E_{r}, E_{\phi}, E_{\theta}$ and $J_{\|}$as a function of $\theta$ for $\phi=\pi / 4, a=0.015 \mathrm{~m}, L_{p}=0.43 \mathrm{~m}$ and $R_{0}=7 \mathrm{~m}$. 


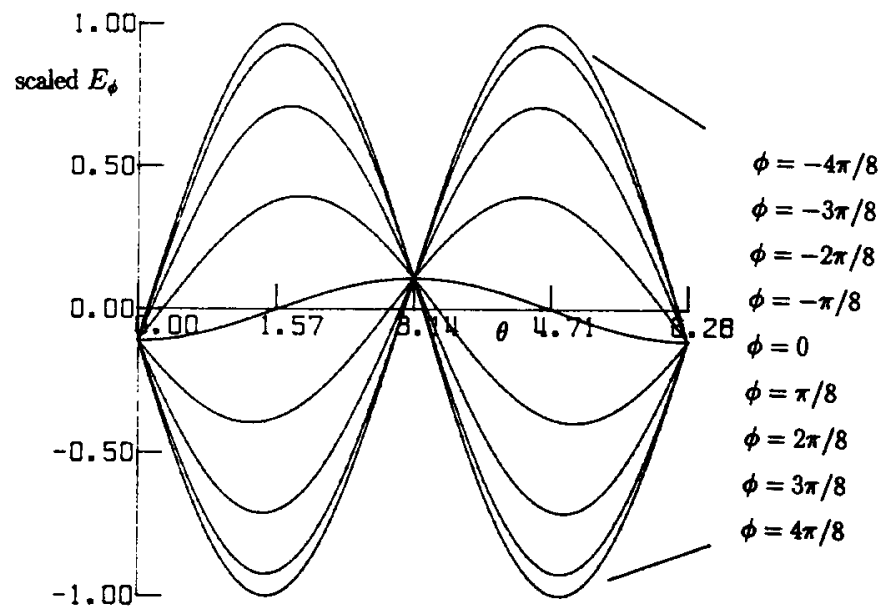

Fig. 6. Scaled value of $E_{\phi}$ as a function of $\theta$ for several values of $\phi . a=0.015 \mathrm{~m}, L_{p}=0.43 \mathrm{~m}$ and $R_{0}=7 \mathrm{~m}$.

direction, both currents proportional to the length of the wire placed in this field. Due to the twisting of the wire, the penetrated flux is linear proportional to the length of the wire in the parallel field. At $\theta= \pm \pi / 2$ the currents are very small compared to the values for $\theta=0$, which means that the current loops have closed via the $r$ direction. This causes high values of $E_{r}$ in these closing regions. A schematic view of the current pattern in the interior if the torus is given in Fig. 7 for small $k$. The surface current is not given but has the same pattern as the bulk current but with opposite sign.

In the Next European Torus reactor configuration $k$ is less than $1 / 100$ which means that $\left|\dot{B}_{z}^{A}\right|$ has to have a very low value for the wire to have unsaturated parts. Furthermore the coupling losses will be high because they are inverse proportional to $k^{2}$. The applied magnetic field on the D-coil largely exceeds our derived upper bound for $\left|\dot{B}_{z}^{A}\right|$ which causes the wire to become saturated if a stationary current pattern is reached. Our analysis does not include screening effects which hold up the penetration of a time varying applied magnetic

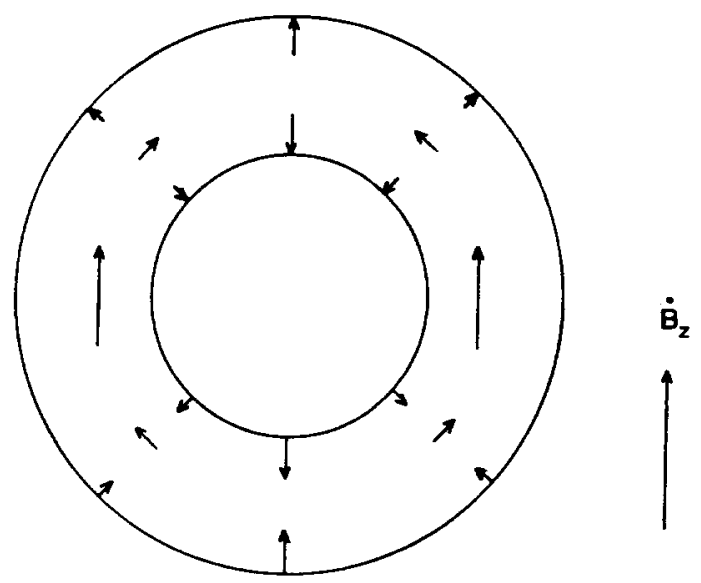

Fig. 7. Schematic view of the current pattern in the interior of the wire for small values of $k$. The surface current (not drawn) has the same pattern as the bulk current but with opposite sign. 
field. Calculations including screening effects have been performed using a straight twisted multifilamentary wire in a parallel field in the $z$ direction $[4,5]$. Turck arrived at a diffusion equation for the current density in the $r$ direction $T_{r}$ :

$$
\frac{\partial^{2} T_{r}}{\partial z^{2}}=\frac{1}{D} \frac{\partial T_{r}}{\partial t},
$$

with $D \approx 2 \times 10^{-4} \mathrm{~m}^{2} / \mathrm{s}$ for a composite superconductor. The analysis is focussed on $T_{r}$, the component causing the singular behaviour of the coupling losses. The diffusion equation results in a time constant $\tau$ for $T_{r}$ of

$$
\tau=\frac{L^{2}}{2 D}
$$

with $2 L$ the length of the wire. Applying this analysis on the torus with $L \approx \pi R_{0} / 2$ would lead to a time constant

$$
\tau \approx \frac{\pi^{2} R_{0}^{2}}{8 D}
$$

For $R_{0}=7 \mathrm{~m}$ the value $\tau \approx 3 \times 10^{5} \mathrm{~s}$ is found, which means that in expected applications no stationary current pattern is reached. Notice that this is a rough estimation of the screening effects, which do not take into account the specific torus shape of the wire. A complete analysis of the time dependent case requires a numerical approach.

\section{Approximation of the bent wire problem}

In the previous section we calculated the solution for the ring shaped wire in the toroidal coordinate system. Another approach for calculating the field and currents in this toroidal configuration is to consider a straight wire and apply a spatially periodic time dependent magnetic field. In the previous sections we have calculated the solution for the torus and therefore it is interesting to compare these two solutions.

The transition from the toroidal to the cylindrical configuration and the definitions concerning this transition are given in Fig. 8. In general we would have to construct the transformation between the $r, \phi, \theta$ system and the $r, \phi, z$ system. We do not perform these calculations but use a different approach as we are only interested in the zero-th order solution in $r$ of the fields and currents. We consider the general form of the applied magnetic field in cylindrical coordinates. A constraint on this form is that the field must be periodic in $z$ with periodicity length $2 \pi R_{0}$. We also take only the lowest Fourier mode in $\phi$ into account. The amplitude of the field parallel and perpendicular to the straight wire can be chosen independently. The reason for applying this strategy is as follows. The cumbersome form of the differential operators in Eqs (33)-(35) is transformed into an easy one, at the cost of a more difficult applied magnetic field.

Now this strategy will be applied in detail. We use Maxwell's equations and conservation of current in cylindrical coordinates. We have to calculate the applied magnetic field in this $r$, $\phi, z$ coordinate system. The field must satisfy 


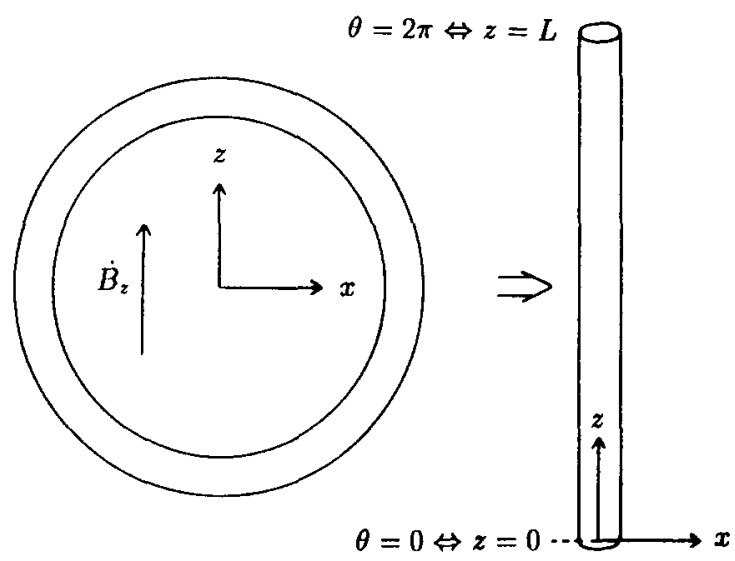

Fig. 8. Transition from the toroidal configuration to the cylindrical configuration.

$\boldsymbol{\nabla} \times \mathbf{B}^{A}=\mathbf{0}$,

$\boldsymbol{\nabla} \cdot \mathbf{B}^{A}=0$.

Basic solutions for this field contain modified Bessel functions in $r$ and Fourier components in $\phi$ and $z$.

Further we need two dominant terms for the applied field:

$$
\begin{aligned}
& \dot{B}_{x}^{A} \approx \dot{B}_{\perp w}^{A} \sin \mu z, \\
& \dot{B}_{z}^{A} \approx \dot{B}_{\| w}^{A} \cos \mu z,
\end{aligned}
$$

while $\dot{B}_{y}^{A} \approx 0$. Here $\mu=2 \pi / L$ and $L=2 \pi R_{0}$, the periodicity length of the applied magnetic field and the subscripts $\perp w$ and $\| w$ stand for perpendicular and parallel to the wire, respectively. The $\dot{B}_{x}^{A}$ and $\dot{B}_{z}^{A}$ term account for the perpendicular and parallel component of the applied magnetic field respectively. For representing the exact torus solution, as given in the previous sections, the applied field has to take into account all the terms in the right hand side of the equations for calculating the terms in the power series of the electric fields. The applied field we derive in this section is only exact for the zero-th order term of the electric fields. The applied field we use is

$$
\begin{aligned}
& \dot{B}_{r}^{A}=2 \dot{B}_{\perp w}^{A}\left[I_{0}(\mu r)-\frac{I_{1}(\mu r)}{\mu r}\right] \cos \phi \sin \mu z+\dot{B}_{\| w}^{A} I_{1}(\mu r) \sin \mu z, \\
& \dot{B}_{\phi}^{A}=-\dot{B}_{\perp w}^{A} \frac{2 I_{1}(\mu r)}{\mu r} \sin \phi \sin \mu z, \\
& \dot{B}_{z}^{A}=\dot{B}_{\| w}^{A} I_{0}(\mu r) \cos \mu z+2 \dot{B}_{\perp w}^{A} I_{1}(\mu r) \cos \phi \cos \mu z .
\end{aligned}
$$

Notice that for $r \rightarrow 0$ this field has an identical form compared with the field given in toroidal coordinates, Eq. (36). The resulting $x$ and $y$ components are

$$
\begin{aligned}
\dot{B}_{x}^{A}= & 2 \dot{B}_{\perp w}^{A}\left[I_{0}(\mu r)-\frac{I_{1}(\mu r)}{\mu r}\right] \cos ^{2} \phi \sin \mu z+\dot{B}_{\perp w}^{A} \frac{2 I_{1}(\mu r)}{\mu r} \sin ^{2} \phi \sin (\mu z) \\
& +\dot{B}_{\| w}^{A} I_{1}(\mu r) \cos \phi \sin \mu z,
\end{aligned}
$$


$\dot{B}_{y}^{A}=\dot{B}_{\perp w}^{A} 2\left[I_{0}(\mu r)-\frac{2 I_{1}(\mu r)}{\mu r}\right] \sin \phi \cos \phi \sin \mu z+\dot{B}_{\| w}^{A} I_{1}(\mu r) \sin \phi \sin \mu z$.

Here $I_{n}(x)$ are modified Bessel functions [11].

The solution for the unsaturated case and no resonances appearing in the system is

$$
\begin{aligned}
E_{r}= & 2 \dot{B}_{\perp w}^{A} c_{1} \frac{I_{1}(\mu r)}{\mu r}[-\cos \phi \sin \mu z+q \sin \phi \cos \mu z]+\dot{B}_{\| w}^{A} \frac{r}{q} I_{0}(\mu r) \sin \mu z \\
& -\dot{B}_{\perp w}^{A} \frac{2 r I_{1}(\mu r)}{1-q^{2}}[-\sin \phi \cos \mu z+q \cos \phi \sin \mu z], \\
E_{\phi}= & 2 \dot{B}_{\perp w}^{A} c_{1}\left[I_{0}(\mu r)-\frac{I_{1}(\mu r)}{\mu r}\right][\sin \phi \sin \mu z+q \cos \phi \cos \mu z] \\
& -\dot{B}_{\| w}^{A} \frac{I_{1}(\mu r)}{\mu} \cos \mu z, \\
E_{z}= & -\frac{2 \pi r}{L_{p}} E_{\phi},
\end{aligned}
$$

with $c_{1}=L_{p} /\left(2 \pi\left(1-q^{2}\right)\right), \mu=2 \pi / L$ and $q=L_{p} / L$. When we use approximations for $I_{0}(x)$ and $I_{1}(x)$ for small $x[11]$ we obtain for the magnetic fields

$$
\begin{aligned}
& \dot{B}_{x}^{A}=\dot{B}_{\perp w}^{A} \sin \mu z+\dot{B}_{\| w}^{A} \frac{\mu r}{2} \cos \phi \sin \mu z+O\left(r^{2}\right), \\
& \dot{B}_{y}^{A}=\dot{B}_{\| w}^{A} \frac{\mu r}{2} \sin \phi \sin \mu r+O\left(r^{2}\right), \\
& \dot{B}_{z}^{A}=\dot{B}_{\| w}^{A} \cos \mu z+\dot{B}_{\perp w}^{A} \mu r \cos \phi \cos \mu z+O\left(r^{2}\right)
\end{aligned}
$$

Note that these expressions include the dominant terms of Eqs (82) and (83). The approximated electric field components are

$$
\begin{aligned}
& E_{r}=\dot{B}_{\perp w}^{A} c_{1}[-\cos \phi \sin \mu z+q \sin \phi \cos \mu z]+\dot{B}_{\| w}^{A} \frac{r}{q} \sin \mu z+O\left(r^{2}\right), \\
& E_{\phi}=\dot{B}_{\perp w}^{A} c_{1}[\sin \phi \sin \mu z+q \cos \phi \cos \mu z]-\dot{B}_{\|_{w}}^{A} \frac{r}{2} \cos \mu z+O\left(r^{2}\right) \\
& E_{z}=-\frac{2 \pi r}{L_{p}} E_{\phi} .
\end{aligned}
$$

We calculate $j_{0}$ using the approximations of the electric field components:

$$
\begin{aligned}
j_{0}= & \frac{L_{p} \sigma_{\perp}}{\pi q^{2}} \dot{B}_{\| w}^{A} \cos \mu z-\frac{2 \pi}{L_{p}} \frac{q\left(1+q^{2}\right)}{1-q^{2}} c_{1} \sigma_{\perp} r \dot{B}_{\perp w}^{A} \cos \phi \cos \mu z \\
& -\frac{2 \pi}{L_{p}} \frac{q^{2}}{1-q^{2}} 2 c_{1} \sigma_{\perp} r \dot{B}_{\perp w}^{A} \sin \phi \sin \mu z+O\left(r^{2}\right) .
\end{aligned}
$$

With the approximations the coupling loss power density in the unsaturated case reads 


$$
\begin{aligned}
P / \mathrm{Vol} & =\frac{\sigma_{\perp}}{\mathrm{Vol}} \int_{0}^{L} \int_{-\pi}^{\pi} \int_{0}^{a}\left(E_{\perp}^{2}+E_{r}^{2}\right) r \mathrm{~d} z \mathrm{~d} \phi \mathrm{d} r, \\
& =\frac{\left(4+q^{2}\right)}{16 q^{2}} a^{2}\left(\dot{B}_{\| w}^{A}\right)^{2} \sigma_{\perp}+\frac{1+q^{2}}{2\left(1-q^{2}\right)^{2}} \frac{L_{p}^{2}}{4 \pi^{2}}\left(\dot{B}_{\perp w}^{A}\right)^{2} \sigma_{\perp}\left[\mathrm{W} / \mathrm{m}^{3}\right],
\end{aligned}
$$

with

$$
\begin{aligned}
& \mathrm{Vol}=\pi L a^{2}, \\
& E_{\perp}=E_{\phi} .
\end{aligned}
$$

The expressions nicely separate the contribution of the parallel field component from that of the perpendicular one. One can see that the singular behaviour for $q \rightarrow 0$ is due to the field component parallel to the wire.

Now we consider $\dot{B}_{\perp w}^{A}=\dot{B}_{\| w}^{A}=\dot{B}_{z}^{A}$. Now recognize that $E_{\phi}^{(0)}$ and $E_{r}^{(0)}$ for the torus solution are exactly represented in $E_{\phi}$ and $E_{r}$ for the straight configuration considered in this section, which should be the case. The terms $E_{\phi}^{(1)}$ and $E_{r}^{(1)}$ are not exactly represented in $E_{\phi}$ and $E_{r}$ for this straight configuration but the terms are identical for $k \rightarrow 0$ and $q \rightarrow 0$. The zero-th order current densities are identical in both cases for $k \rightarrow 0$ and $q \rightarrow 0$. Notice that for $k \rightarrow 0$ and $q \rightarrow 0$ the losses for the torus and this straight wire are identical.

It can be concluded that the torus problem can be approximated by a straight wire in a spatially dependent magnetic field for small values of $k$. Furthermore the expansion of the magnetic field in a Fourier series, as was mentioned in the introduction, can best be performed in this straight wire configuration.

\section{Conclusion}

In case of a stationary solution, the analytical analysis of a multifilamentary, superconducting, twisted, torus shaped wire in a time varying magnetic field can be performed. Expressions for the electric field and currents have been obtained.

Basic assumptions for the shown analysis are:

1. the problem is stationary;

2. no saturation occurs (the problem is linear);

3. Carr's anisotropic continuum model can be applied;

4. the shielding currents at the surface of the wire can be taken into account using a (linear) surface current, which flows in the filament direction;

5. the radius of the wire $a$ is very small compared to the mean radius of the torus $R_{0}$ and the twist length $L_{p}$ is less than $2 \pi R_{0} ; k=L_{p} /\left(2 \pi R_{0}\right)$ is an irrational number;

6. the solution can be written as a series expansion in $r$.

Higher Fourier modes appear in the $\phi$-dependence of the solution because $h_{\theta}$ contains the term $r \cos \phi$. As long as the wire is unsaturated the problem is linear which means that for calculating the solution of complicated field patterns the superposition principle can be used.

For small values of $k$ the coupling losses increase inversely proportional to $k^{2}$ whereas the rate of change of the magnetic field must be small for the wire to be unsaturated. $E_{\phi}$ is bounded but $E_{r}$ and the bulk and surface current are singular. This is caused by the parallel 
magnetic field component. The current loops close via the $r$ direction causing high values of $E_{r}$ in these closing regions. These properties can cause severe complications for the use of superconducting wires in the Next European Torus fusion reactor. An important extension of the problem would be the calculation of the time dependent response. Furthermore, for small values of $k$ the torus problem can be approximated by a straight wire in a spatially dependent magnetic field.

\section{Acknowledgment}

This work is part of a research program for NET.

\section{References}

1. P.C. Rem, Numerical models for ac superconductors. Thesis, University of Twente, June 1986.

2. D. Ciazynski and B. Turck, Theoretical and experimental study of the saturation of a superconducting composite under fast changing magnetic field. Cryogenics 24 (1984) 507-514.

3. R.A. Hartmann, A contribution to the understanding of ac losses in composite superconductors. Thesis, University of Twente, Sept. 1989.

4. B. Turck, Courants de circulation et pertes dans les composites supraconducteurs soumis a une induction longitudinale variable. Revue de physiquee appliquée (1976) 369.

5. G. Ries and K.P. Jüngst, Filament coupling in multifilamentary superconductors in pulsed longitudinal fields. Cryogenics (March 1976).

6. Fusion Technology 14 (1988).

7. H. London, Alternating current losses in superconductors of the second kind. Phys. Rev. Lett. 6 (1963) $162-165$.

8. C.P. Bean, Magnetization of hard superconductors. Phys. Rev. Lett. 8 (1962) $250-253$.

9. W.J. Carr, Jr., Electromagnetic theory for filamentary superconductors. Phys. Rev. B11 (1975) 1547-1554.

10. P.M. Morse and H. Feshbach, Methods of Theoretical Physics. New York: McGraw-Hill (1953).

11. M. Abramowitz and I. Stegun, Handbook of Mathematical Functions. New York: John Wiley \& Sons (1972). 